\title{
Cold Gas Reaction Control System for the Near Earth Asteroid Scout CubeSat
}

\author{
Brandon C. Stiltner ${ }^{1}$ and Ben Diedrich. ${ }^{2}$ \\ Jacobs ESSSA Group / NASA MSFC, Huntsville, AL, 35812 \\ Chris Becker ${ }^{3}$, Ivan Bertaska ${ }^{4}$, Andrew Heaton $^{5}$, and Juan Orphee ${ }^{6}$ \\ NASA MSFC, Huntsville, AL, 35812
}

This paper describes the Attitude Control System (ACS) for the Near Earth Asteroid (NEA) Scout cubesat with particular focus on the Reaction Control System (RCS). NEA Scout is a $6 \mathrm{U}$ cubesat with an 86 square-meter solar sail. NEA Scout will launch on Space Launch System (SLS) Exploration Mission 1 (EM-1), currently scheduled to launch in 2019. The spacecraft will rendezvous with an asteroid after a two year journey, and will conduct science imagery. The ACS consists of three major actuating subsystems: a Reaction Wheel (RW) control system, a Reaction Control System (RCS), and an Active Mass Translator (AMT) system. The three subsystems allow for a wide range of spacecraft attitude control capabilities, needed for the different phases of the NEA-Scout mission. The RCS performs a number of critical functions during NEA Scout's mission. These requirements are described and the performance for achieving these requirements is shown. Moreover, NEA Scout employs a solar sail for long-duration propulsion. Solar sails are large, flexible structures that typically have low bending-mode frequencies. This paper demonstrates a robust performance while avoiding excitation of the sail's structural modes.

$\begin{array}{ll}\text { Nomenclature } \\ \theta & =\text { generic symbol for attitude angle (Gibbs vector definition) about an arbitrary axis } \\ \theta^{*} & =\text { phase-plane attitude deadband, tunable control parameter } \\ \theta_{d} & =\text { phase-plane drift line inflection point where drift lines become sloped lines } \\ \omega^{*} & =\text { generic symbol for angular rate about an arbitrary axis } \\ \omega^{*} & =\text { phase-plane angular rate deadband, tunable control parameter } \\ \omega_{d} & =\text { phase-plane drift line used to define the drift region. } \\ \omega_{d o} & =\text { phase-plane outer drift line that defines the upper bound of the drift region }\end{array}$

\section{Introduction}

The Near Earth Asteroid (NEA) Scout spacecraft is a 6U cubesat that is currently under development by NASA as a joint effort between NASA Marshall Space Flight Center (MSFC) and the Jet Propulsion Laboratory (JPL). NEA Scout will launch on Space Launch System (SLS) Exploration Mission (EM) 1, currently scheduled to launch in 2019. NEA Scout's primary mission objective is to rendezvous with a near Earth asteroid and perform science imagery. The baseline asteroid for mission planning is known as 1991 VG [1], however several other asteroids are also under consideration to account for different launch dates. Specifically, NEA Scout's scientific objectives are to better characterize the target asteroid's orbit, and to take pictures of its surface. This paper discusses the overall Attitude Control System (ACS) for NEA Scout. Specific attention is given to the Reaction Control System (RCS), which uses a cold-gas propellant for attitude control.

\footnotetext{
${ }^{1}$ GN\&C Engineer, Jacobs ESSSA Group / NASA MSFC, AIAA Senior Member.

${ }^{2}$ GN\&C Engineer, Jacobs ESSSA Group / NASA MSFC.

${ }^{3}$ GN\&C Engineer, NASA MSFC.

${ }^{4}$ GN\&C Engineer, NASA MSFC.

${ }^{5}$ Senior Aerospace Engineer, NASA MSFC, AIAA Senior Member.

${ }^{6}$ GN\&C Engineer, NASA MSFC, AIAA Member.
} 
The remainder of this paper is organized as follows. Section II provides an overview of the NEA Scout cubesat and its mission. Section III provides an overview of ACS hardware, which discusses the primary control actuators and sensors. This section provides an in depth overview of the RCS hardware. Section IV details the RCS responsibilities and control system design. Section V details the RCS performance against the requirements listed in Section IV. In Section VI, a brief summary of solar sail flex-dynamics is provided, along with how the RCS is designed to avoid exciting these dynamics. Last, remaining conclusions are provided in Section VII.

\section{NEA Scout Overview}

The NEA Scout spacecraft and mission have both been described in several publications $[2,3,4]$. NEA Scout is a $6 \mathrm{U}$ cubesat that will use an $86 \mathrm{~m}^{2}$ solar-sail for propulsion. An image of the NEA Scout cubesat is shown in Figure 1 with the solar sail in its stowed configuration. In the image, most of the ACS hardware is called out. As shown, there are 3 primary control actuators on NEA Scout: the Reaction Wheel (RW) control system, the RCS, and the Active Mass Translator (AMT). The functions of each control actuator are discussed in Section III. An overview of the NEA Scout control system is also provided in Orphee et al. [5]. Also shown are the attitude determination sensors: three coarse sun sensors, an Inertial Measurement Unit (IMU), and a star tracker. These devices are also described in Section III.

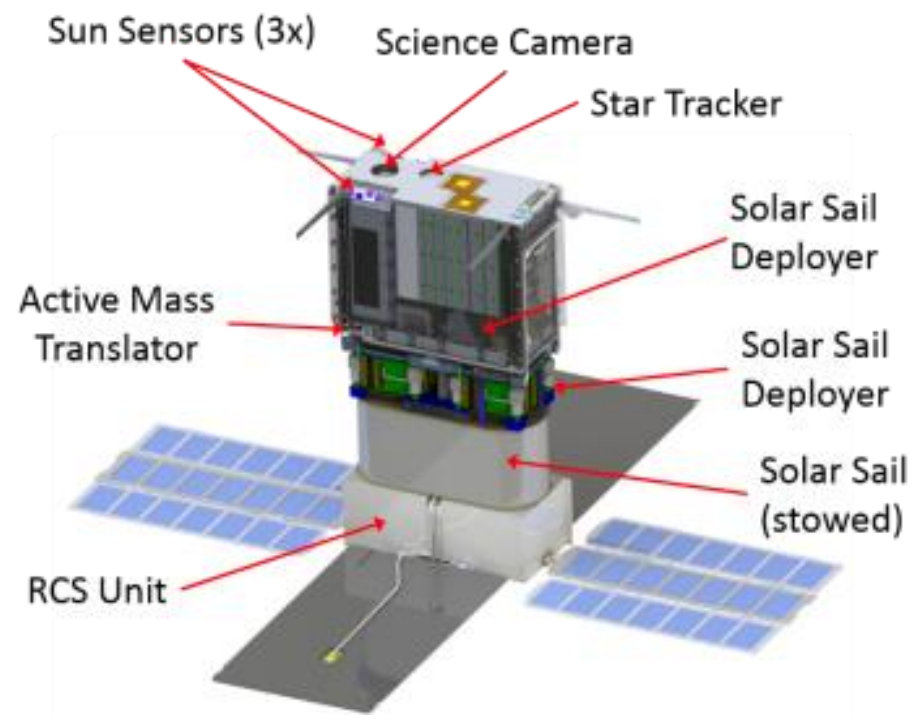

Figure 1: CAD image of NEA Scout showing the spacecraft in two different orientations and calling out the names and locations of various spacecraft components.

NEA Scout is divided into three major pieces as shown in Figure 2. Starting at the bottom, the solar panels are attached to the RCS unit. The RCS unit occupies approximately $2 \mathrm{U}$ of the spacecraft's volume and is outlined in green. In the middle $2 \mathrm{U}$ are the solar sail and sail deployer outlined in blue. The portion outlined in orange is the spacecraft's avionics bus which occupies the upper $2 \mathrm{U}$ of volume. This portion contains the science camera, star tracker, flight computer, reaction wheel assembly, and other avionics. For the majority of NEA Scout's mission, this side of the spacecraft will be pointed away from the sun and will be in the shadow of the solar sail. Figure 3 provides an image of NEA Scout before and after the solar sail is deployed. The left of the figure also shows NEA Scout's coordinate frame with the $\mathrm{Z}$-axis pointing in a direction normal to the solar panels and sail. 


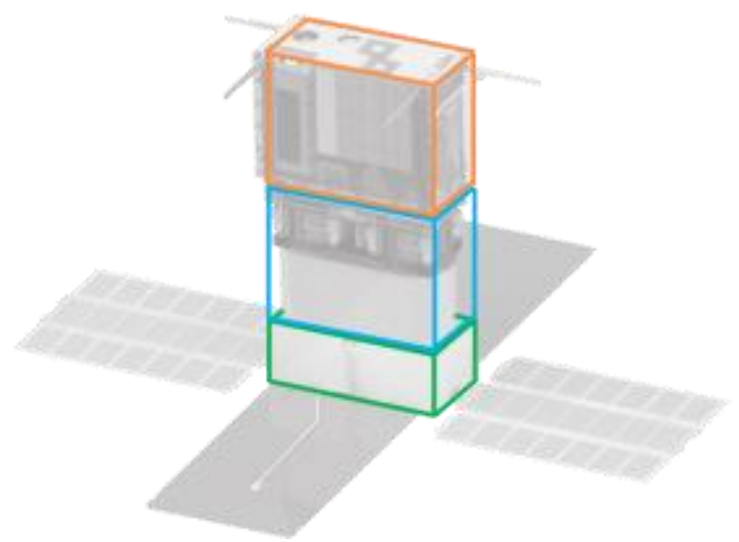

Figure 2: CAD image of NEA Scout calling out the three major pieces of the spacecraft: RCS unit (green), solar sail and deployer mechanism (blue), and avionics bus (orange).

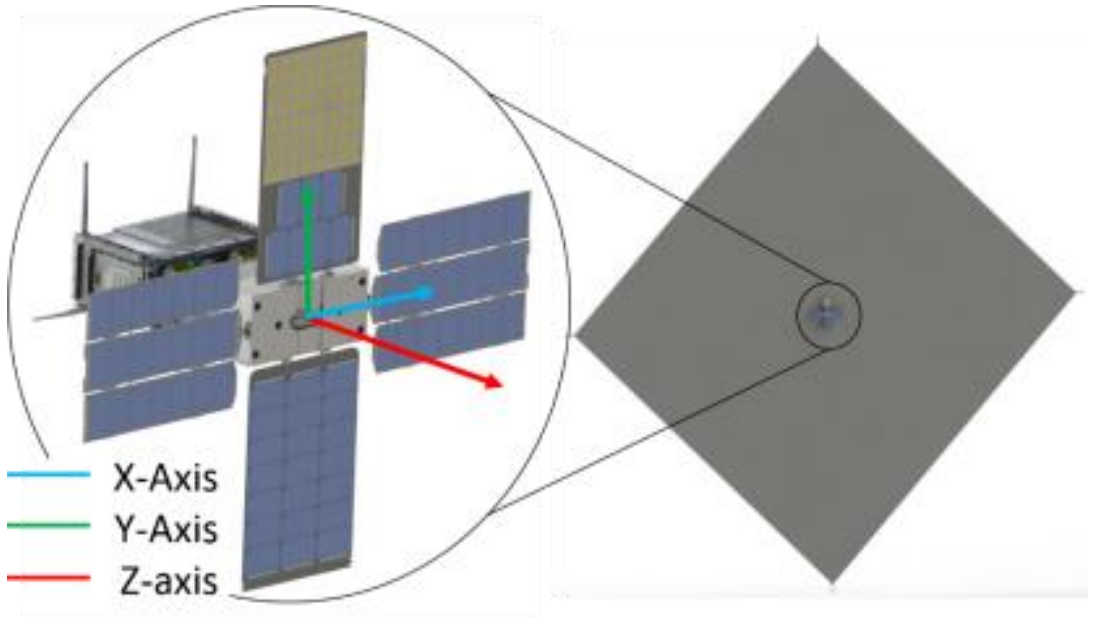

Figure 3: (left) Image of NEA Scout before solar sail deployment and (right) NEA Scout after solar sail deployment. The grey diamond on the right is the deployed solar sail, which is $86 \mathrm{~m}^{2}$ in size.

\section{A. NEA Scout Mission Overview}

\section{Pre-Mission, Launch, and Ejection from SLS}

As mentioned above, NEA Scout will launch on SLS EM 1 in 2019. Prior to launch, it will in storage for up to a year before being loaded onto SLS. NEA Scout, along with thirteen other CubeSats, is stowed on SLS as shown in Figure 4. This portion of SLS is between the Orion Multi-Purpose Crew Vehicle (MPCV) and the Interim Cryogenic Propulsion System (ICPS) upper stage. The CubeSats are located around the circumference of the MPCV Stage Adapter (MSA).

After launch, ICPS and MPCV are placed into an elliptic checkout orbit around Earth. During this time, various systems are checked on MPCV to ensure they are functioning properly before beginning the trip to the moon. After system checks, the ICPS performs a propulsive maneuver known as the Trans-Lunar Injection (TLI). The TLI maneuver places MPCV and ICPS on a trajectory to the moon. MPCV and ICPS separate shortly after the TLI burn, and the MSA remains attached to the ICPS. Finally, the ICPS performs its disposal activities, depleting all remaining propellants and batteries. The propellant depletion provides some additional $\boldsymbol{\Delta} \boldsymbol{V}$ to ICPS and also targets a lunar flyby that will provide a lunar gravity assist into a heliocentric orbit.

Shortly after ICPS' disposal activities, the first round of CubeSats are ejected. The CubeSats are ejected in batches to minimize the chances of colliding with each other. Each ejection opportunity is known as a bus stop, and the schedule of bus stops is depicted in Figure 5. NEA Scout is scheduled to eject during the first bus stop. 


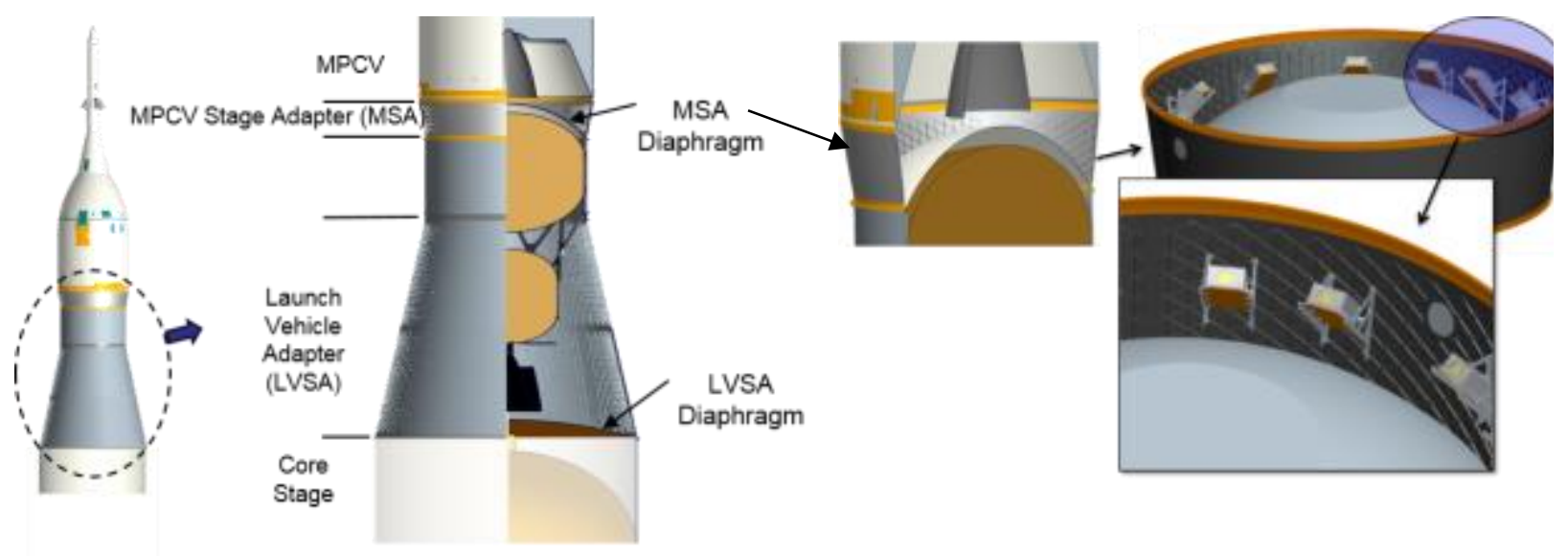

Figure 4: (Left) Image of the SLS showing the upper stage (ICPS), MSA, and MPCV. (Right) Three detailed views of the MSA and CubeSat payloads located along the circumference of the MSA.

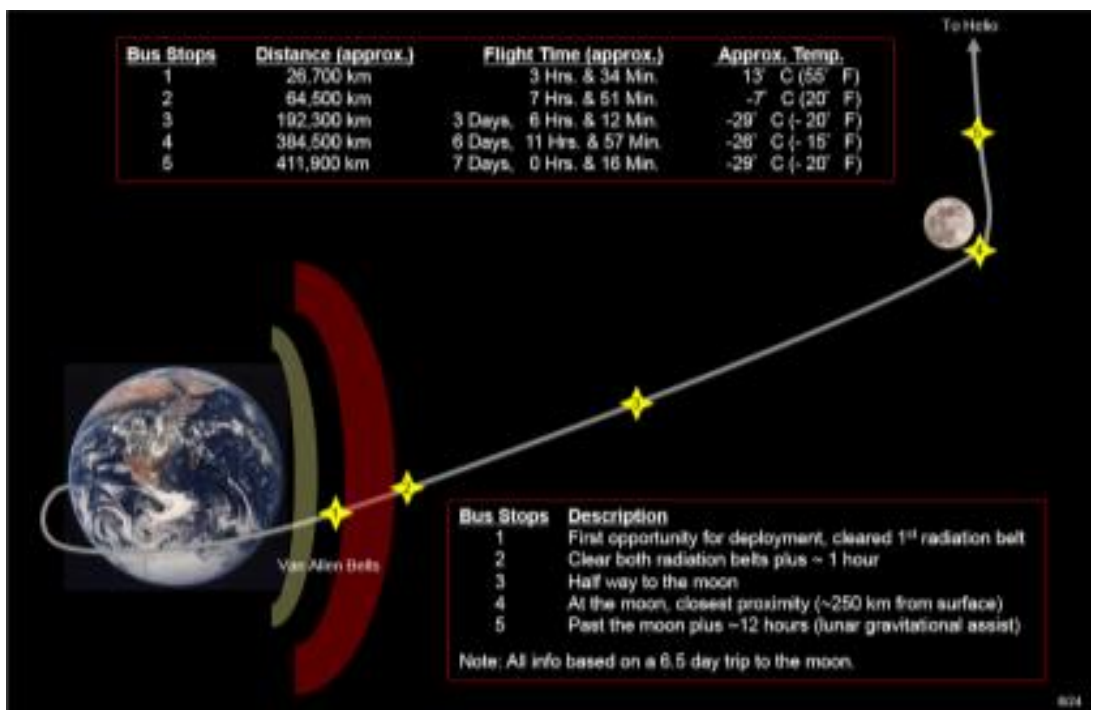

Figure 5: Graphic depicting the ICPS trajectory and scheduled bus stops for CubeSat deployment.

\section{Early Mission}

Immediately after ejection, NEA Scout must first perform a detumble maneuver. This maneuver is performed by the RCS and IMU, and nulls any angular rates imparted onto the spacecraft during ejection. After detumble, NEA Scout performs an autonomous sun-finding maneuver using the RCS, IMU, and sun sensors. NEA Scout will have been in a powered-off state for months prior to ejection from SLS. Because of this, NEA Scout will not have an accurate onboard clock, and therefore cannot use onboard ephemeris data to point to the sun. Instead, it must use its sun sensors to locate and point toward the sun. Once correctly pointing at the sun, it deploys solar arrays to begin charging its batteries.

NEA Scout remains in a sun pointing attitude until the first communications opportunity. At this time, data is downloaded from NEA Scout and scripted activities are uploaded into NEA Scout's non-volatile memory. Also, the onboard clock and onboard ephemeris are updated, allowing for more accurate pointing toward the sun. Furthermore, this opportunity is used for system checkout, calibration, and overall health and status of NEA Scout. A navigation update is also performed during this time, which allows engineers on the ground to plan the Trajectory Correction Maneuver (TCM).

The TCM is a corrective propulsive $(\boldsymbol{\Delta V})$ maneuver that uses the RCS axial jets for propulsion. The RCS axial jets are described in Section III.A. As the name implies, the TCM corrects NEA Scout's trajectory by targeting a particular lunar fly-by for a lunar gravity assist. The maneuver may require a total $\boldsymbol{\Delta} \boldsymbol{V}$ of up to $17 \mathrm{~m} / \mathrm{s}$ to achieve the correct lunar fly-by, and will be performed in segments due to propellant heating and power constraints. This maneuver is discussed in more detail in Section IV.A. 
The first lunar fly-by does not provide enough energy to escape the Earth-Moon system. So, NEA Scout will make another Earth fly-by, and a second lunar fly-by to achieve the desired heliocentric orbit. NEA Scout deploys its solar sail after the first lunar fly-by and begins solar sailing during this early phase of the mission. In total, NEA Scout will spend approximately 1.5 months in the Earth-Moon system.

\section{Solar Sailing, Science Mission, and End-of-Life}

Once in a heliocentric orbit, NEA Scout could spend up to 2.5 years flying toward its target, and its design life is four years. At present, NEA Scout's mission is based on the Near Earth Object 1991 VG. However, several other asteroids are under consideration, and the actual asteroid will be selected based on SLS' particular launch date. While in route, NEA Scout will use its solar sail to maneuver toward the asteroid. It will perform weekly communications slews where solar-sail attitude updates are uploaded. As it draws near its target, NEA Scout will do preliminary searches for the asteroid. These will be done to recognize the asteroid and to allow for navigation updates to target a close fly-by of the asteroid.

At present, NEA Scout is targeting a fly-by not to exceed 750 meters at closest approach. The minimum distance may be closer and will depend on the mission geometry and resulting orbits of the asteroid and NEA Scout. During the rendezvous, NEA Scout will point its science camera toward the asteroid and will perform a Y-axis slew to keep the asteroid in the camera's field of view throughout the fly-by. During the fly-by, images will be taken of the asteroid in the visible spectrum. The science objectives are to characterize the asteroid's orbit, rotational dynamics, and surface regolith. After the fly-by, NEA Scout will have accomplished all of its primary mission and science objectives.

Depending on the actual time required to rendezvous with the asteroid, NEA Scout may still have some life span remaining. For example, the baseline mission targeting $1991 \mathrm{VG}$ will result in a 2.5 year duration, leaving NEA Scout with an additional 1.5 years of lifespan. If this case should occur, NEA Scout will perform one of several secondary objectives. Those include attempting a second rendezvous with the primary target asteroid, or attempting to rendezvous with another asteroid. The primary mission duration depends on a number of factors. In particular, the launch date and resulting primary target. NEA Scout's design lifespan is four years, not including time spent in storage on Earth prior to launch.

\section{NEA Scout ACS Overview}

\section{A. Attitude Control Effectors}

\section{1. $R C S$}

The RCS unit is shown in Figure 1 and Figure 2. The unit occupies approximately $2 \mathrm{U}$ of volume on NEA Scout and contains $1.25 \mathrm{~kg}$ of propellant when full. The propellant is refrigerant R236fa. A conceptual image of the RCS unit is shown in Figure 6. The four circular features at the corners represent the attitude control jets, and arrows are used to depict the direction of thrust for each jet. The attitude control jets are arranged so that firing any pair generates torque about one of the spacecraft body axes. For example, firing jets 1 and 2 create a positive $Y$-axis torque, and jets 2 and 4 create a positive Z-axis torque. The two circular features in the center of the RCS unit are the axial jets, with force components along the negative z-axis (into the page). The RCS unit is being manufactured by VACCO industries ${ }^{7}$.

\footnotetext{
${ }^{7}$ http://www.vacco.com/
} 


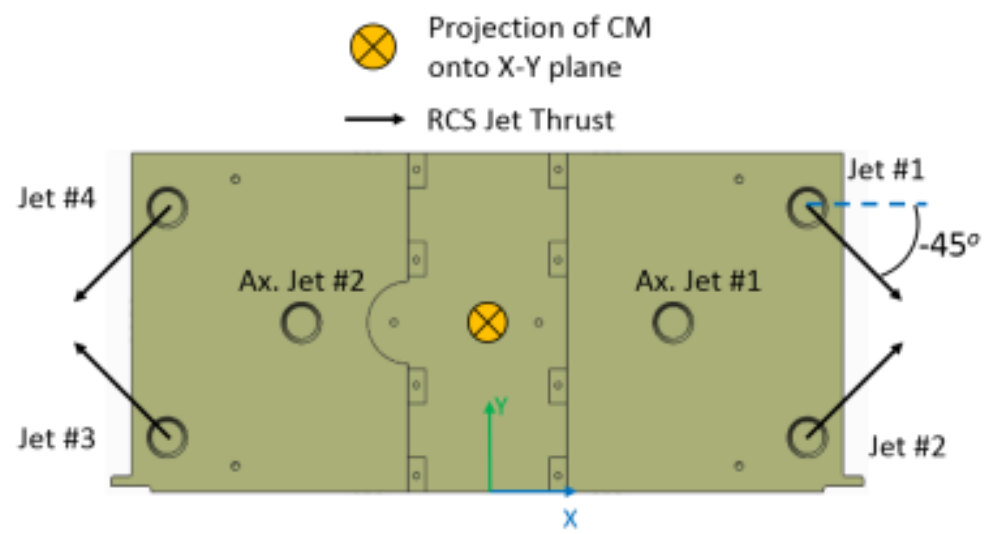

Figure 6: Conceptual image of the RCS unit showing the four attitude control jets and two axial jets. The force directions are visualized as black arrows, and the projection of the spacecraft Center of Mass (CM) is also shown on the RCS unit.

\section{Reaction Wheel System}

The Reaction Wheel (RW) hardware is composed of four reaction wheels with $0.015 \mathrm{~N}-\mathrm{m}$-s capacity each. The wheels are arranged in a pyramid and each spin axis is canted approximately 60 degrees from the body Z-axis. Each wheel is also canted about 45 degrees relative to the body $\mathrm{X}$ and $\mathrm{Y}$ axes. This arrangement allows for redundancy in case any one of the reaction wheels fail. The reaction wheels serve as the primary attitude control actuator throughout most of NEA Scout's mission. Due to their small size, they are used for fine pointing and control of the spacecraft. However, as with all reaction wheels, they are subject to momentum buildup. The RCS and AMT are used to desaturate the momentum wheels during the mission as described in Section IV.D. The RW control logic outputs a command torque about the three body axes. This torque is decomposed by an allocation matrix that distributes the torque to each reaction wheel. The algorithm uses a Moore-Penrose pseudo inverse to compute the torque command for each wheel. In case of a single reaction wheel failure, the RCS takes over control of NEA Scout until a new allocation matrix can be uploaded from the ground. The reaction wheels are from Blue Canyon Technologies ${ }^{8}$.

\section{Active Mass Translator (AMT)}

The Active Mass Translator (AMT) is a mechanism that moves the center of mass (CM) of the spacecraft relative to the center of pressure (CP) of the solar sail. The AMT functions similarly to a milling table, allowing for displacement in the $\mathrm{X}$ and $\mathrm{Y}$ axes. The mechanism is shown on the right of Figure 7 with displacement along the negative $\mathrm{X}$ axis. The solar sail deployer (blue and green components) and solar sail spool (gold and dark grey components) are shown attached to the AMT. The solar sail is in its stowed configuration in this image. On one side of the AMT is the spacecraft avionics bus. On the other side is the solar sail, RCS unit, and solar panels. The AMT effectively splits the spacecraft in half, and allows the two halves to move relative to one another. In doing so, NEA Scout's center of mass is shifted in the $\mathrm{X}$ and $\mathrm{Y}$ body axes. Due to imperfections in the deployment and other effects, solar sails typically generate a small disturbance torque on the spacecraft. This is referred to as solar torque. The CM movement caused by the AMT is enough to change the direction of solar torque in both the $\mathrm{X}$ and $\mathrm{Y}$ body axes. This allows the spacecraft to utilize the solar torque to reduce the reaction wheel momentum accumulation, and those torques can also be trimmed. Z-axis momentum accumulation is controlled separately through a mixture of solar sail attitude and RCS.

\footnotetext{
${ }^{8}$ http://bluecanyontech.com/reaction-wheels/
} 


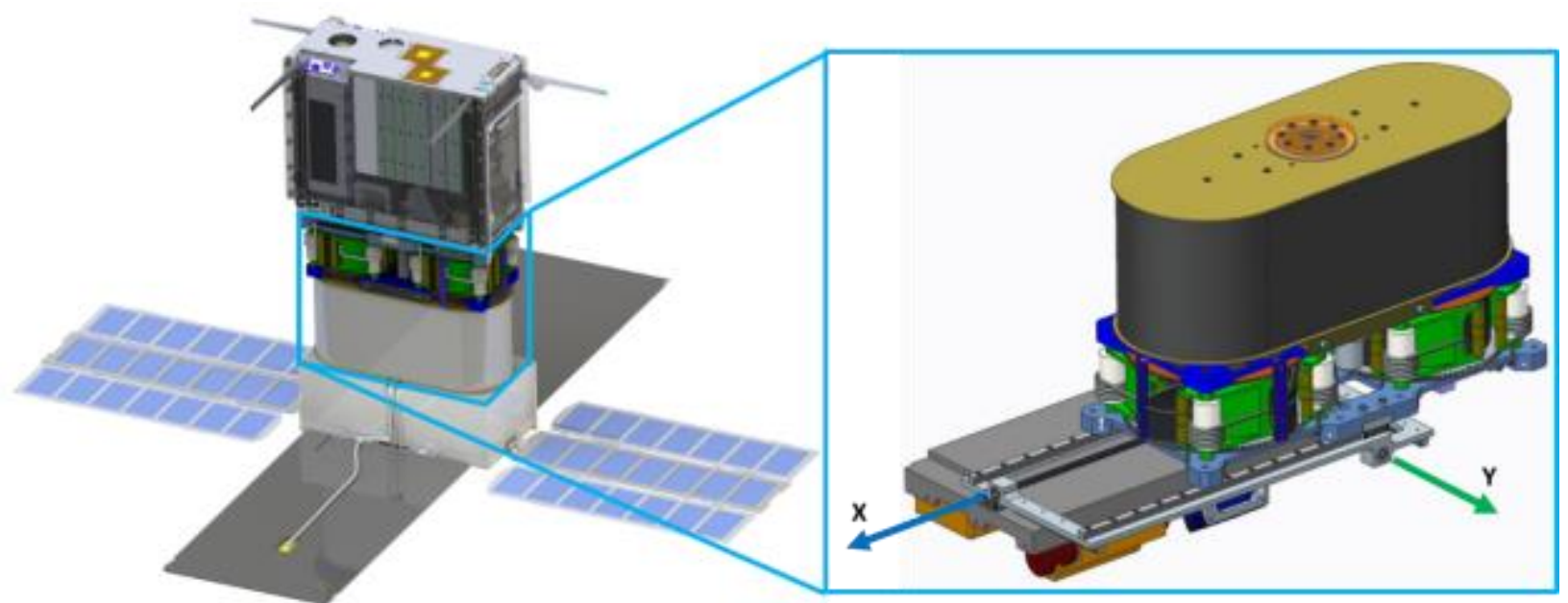

Figure 7: The Active Mass Translator (AMT) displaces the avionics bus relative to the solar sail and RCS unit, shifting the spacecraft's center of mass relative to the center of pressure of the sail. (From left to right, components have been flipped and rotated in the image).

\section{B. Attitude Determination Sensors}

\section{Sun Sensors}

NEA Scout has three sun sensor units from Blue Canyon Technologies. Two sun sensor units are located on the avionics bus, and the third is located on the RCS as shown in Figure 1. Each unit consists of four photodiodes arranged in a pyramid-like orientation. Each photodiode provides a value known as an illumination count. The values are largest when a diode is directly facing the sun, and smaller when inclined or facing away from the sun. The sun sensors are calibrated from on-orbit testing performed by the manufacturer.

The sun sensors are primarily used for initial sun pointing after NEA Scout is ejected and during safe mode operations. Because these sensors provide a low accuracy attitude solution, they are used very little throughout the bulk of NEA Scout's mission. However, their use in initial sun pointing is crucial. Upon initial boot up, NEA Scout will not have an accurate onboard clock, so it cannot locate the Sun using onboard ephemeris data. Therefore, the sun sensors are used to initially orient the spacecraft towards the sun for battery charging. To do so, NEA Scout will perform an autonomous sun finding routine. If the Sun is initially in view of one of the sun sensors, a relative attitude quaternion is determined, allowing the spacecraft to slew towards the Sun. If no sun sensors are in view of the Sun, a sun finding search pattern is used to locate the Sun. Because of the location of three sun sensors on the spacecraft, a full sky search for the Sun is performed by first performing a ninety degree slew about the spacecraft Y-axis followed by a ninety degree slew about the $\mathrm{X}$-axis. Once the Sun is found, the search pattern is terminated and the spacecraft is oriented towards the Sun for charging.

\section{IMU}

A Sensonor STIM $300^{9}$ Inertial Measurement Unit (IMU) was selected for NEA Scout due to its small form-factor and sufficient noise properties. Although the IMU is equipped with an accelerometer, gyroscope, and an inclinometer, only the gyroscope measurements (i.e., body angular rates) are used in the attitude determination and control system. The STIM300 utilizes a three-axis Micro-Electro-Mechanical System (MEMS) gyroscope, whose output is filtered through a cascaded-integrator-comb (CIC) filter operating at $2000 \mathrm{~Hz}$. The cutoff frequency for the CIC filter is userselectable. The output is additionally filtered through an eighth-order Butterworth low-pass filter before being incorporated into the attitude solution for the guidance and control $(\mathrm{G} \& \mathrm{C})$ software. Due to the requirement of small body rates while the solar sail is deployed, a star tracker is used as the primary rate sensor for most of NEA Scout's mission. The STIM300 is used during the early mission phase, and as a backup in times when the spacecraft attitude prevents the star tracker from acquiring a solution (i.e., Sun/Earth/Moon blinding). In these cases, the IMU body rate measurements are propagated for the duration of the blinding event.

A challenge in designing the control architecture around the IMU gyroscopes is in the unusually long 'shelf-life' of NEA Scout - the period of time between final testing and ejection from SLS. MEMS gyroscopes are susceptible

\footnotetext{
${ }^{9}$ http://www.sensonor.com/gyro-products/inertial-measurement-units/stim300.aspx
} 
to static charging affects, which can cause drift in the gyroscope biases even when the unit is powered off. A statistical analysis was performed on a history of STIM300 units to adequately determine the risk imposed by static charging.

\section{Star Tracker}

The Blue Canyon Technologies (BCT) Thin-Slice Nano Star Tracker (NST) ${ }^{10}$ is the primary attitude and rate sensor onboard NEA Scout. The star tracker provides both inertial attitude and body angular rates. The angular rates are computed by backward differencing of the current and previous attitude quaternions. To determine the attitude, a star field in view is compared to the onboard star catalogue. The star tracker outputs the attitude and angular rates at $5 \mathrm{~Hz}$. An eighth-order Butterworth low-pass filter is used to filter the angular rate measurements before being used in the G\&C software. The attitude solution from the star tracker is used as the primary measurement for spacecraft attitude. If the star tracker suffers a blinding event, the IMU is used as a backup by propagating the angular rates to obtain an attitude solution.

The Thin-Slice NST is a modified version of the BCT Standard Nano Star Tracker (NST) that was designed to accommodate NEA Scout's volume constraints. A substantial portion of the Standard NST baffle was removed to fit an allotted volume for both the star tracker optics and control card. This increased the keep-out zones associated with sun occlusion and Earth albedo occlusion (45/25 degrees for sun/Earth keep-out for the Standard NST, compared with 180/100 degrees for sun/Earth keep-out for the Thin-Slice NST). This imposed requirements on mission design, and necessitated substantial analysis of the effect from glint off the solar sail onto the star tracker optics.

\section{RCS Responsibilities and Design}

\section{A. RCS Responsibilities}

NEA Scout uses a cold gas RCS to control the spacecraft's attitude at various times during the mission. Specifically, the RCS has five responsibilities:

- Initial spacecraft detumble

- Initial sun-pointing and attitude hold

- Trajectory Correction Maneuver (TCM)

- Reaction wheel momentum desaturation

- Safe mode operations

NEA Scout will be ejected from SLS with some residual angular rates (up to 16 degrees per second on each body axis). The spacecraft power state will also be unknown, as the vehicle could be in storage for up to a year prior to SLS launch. Therefore, the operations immediately following ejection are to null the angular rates and point toward the sun to charge the batteries. After achieving a positive charging state, the reaction wheels will take over as the primary actuator for the spacecraft.

Throughout NEA Scout's mission, attitude control is handed over to the RCS for certain operations. One example is during the TCM. This maneuver is performed to achieve a desired lunar fly-by, and occurs within hours of the ejection from SLS. Here, the axial jets fire continuously to provide the necessary $\boldsymbol{\Delta} \boldsymbol{V}$, while the RCS jets maintain the spacecraft's attitude during the maneuver. Attitude control is handled by the RCS during the TCM because the torques would saturate the wheel speeds before the TCM concluded.

Any time the reaction wheels are active, the RCS remains active to desaturate the reaction wheel momentum. Furthermore, the RCS is also used for any safe-mode operations, like attitude recovery. Safe-mode operations are those that occur due to unexpected events, such as a flight computer reboot or other unforeseen problem that occurs during flight. At the time of writing this paper, the NEA Scout safe-mode is currently being defined, but will likely be an attitude recovery and maneuver to point-and-hold the solar panels toward the sun.

\section{B. Phase-plane Control Design}

NEA Scout uses simple logic known as a phase-plane control system for the RCS. This type of control is sometimes referred to as a Schmitt Trigger $[6,7,8]$ or a bang-off-bang controller. Phase-plane controllers have been used extensively as the control logic for spacecraft RCS. In particular, the Space Shuttle utilized a phase-plane controller [8]. A phase-plane controller is best described visually as shown in Figure 8. The figures show a Cartesian coordinate frame with the attitude error on the $\mathrm{x}$-axis $\left(\theta_{\mathrm{e}}\right)$ and angular rate error on the $\mathrm{y}$-axis $\left(\omega_{\mathrm{e}}\right)$. Error is defined as the difference between measured and commanded values. The symbol $\theta$ is used to generically denote attitude, and should not be confused with the traditional Euler angle usage. The attitude error is converted from a quaternion into a Gibbs vector attitude description [7]. In this way, the attitude is reduced from four values to three, each

\footnotetext{
${ }^{10} \mathrm{http}: / /$ bluecanyontech.com/thin-slice-nst/
} 
corresponding to angular displacement about the spacecraft body axes. The angular rate error is simply the difference of the commanded and measured body axis angular rates. Red lines denote the switching lines, where the horizontal lines define the drift regions. The grey inner-portion denotes the quiescent region, or deadband. If the attitude and rate errors fall within the deadband, control is left off. Otherwise, the control is on and the system is driven toward the origin of the phase-plane.
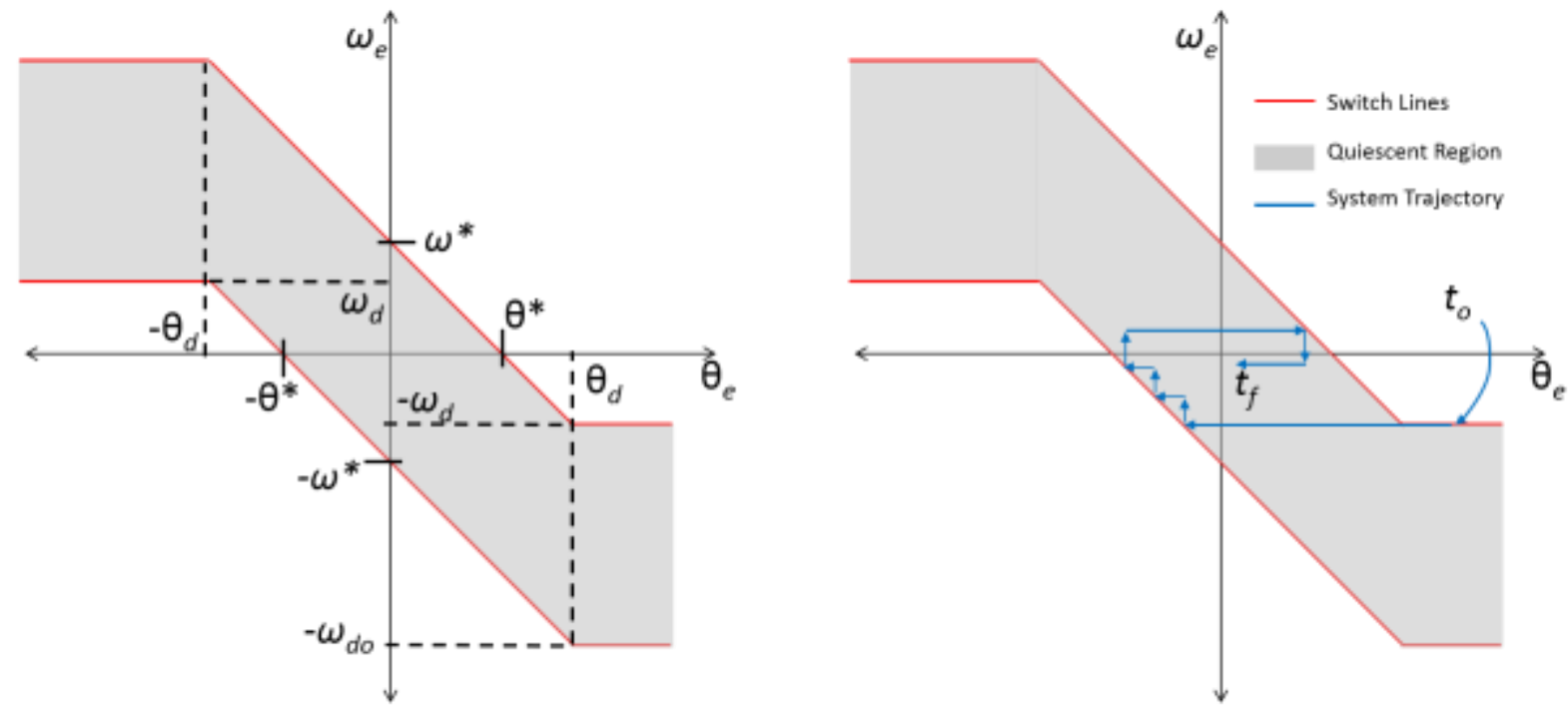

Figure 8: (left) A theoretical phase-plane diagram showing the tunable parameters that define the switching lines. (right) Illustration of the concept of the switching lines and the deadband using blue arrows to depict a theoretical system trajectory subject to a phase-plane control system.

On NEA Scout, each body axis has an individual phase-plane. Therefore, the angular rate and attitude errors are evaluated for each body axis. If the values are outside the deadband (outside the grey region), a pair of RCS jets are opened to drive the system toward the deadband. Jet selection is discussed in Section IV.B.2. On NEA Scout, the phase-planes are defined with three parameters: $\omega^{*}, \theta^{*}$, and $\omega_{d}$. These values are shown on the left of Figure 8 , and are detailed in the following subsection.

A theoretical system trajectory is shown on the right of Figure 8, depicted with blue arrows. At $t_{0}$, the rate and attitude errors are outside the deadband, so a pair of RCS jets are opened to apply a corrective torque. These jets remain open on consecutive iterations of the flight software until reaching a switching line. As shown in the figure, the system trajectory moves into the $4^{\text {th }}$ quadrant of the phase-plane and reaches a drift line. At this point, the jets are switched off and the system is quiescent. However, because the angular rate error is non-zero, the system's attitude error drifts across the deadband until reaching the sloped line. The system follows a staircase path along the switching line caused by opening and closing the jets. This effect is the result of a digital (non-continuous) control system. Once the angular rate error is positive, the attitude drifts back across the deadband toward the opposite switching line. If there are no disturbance torques on the vehicle, this system will continue to encircle the origin of the phase-plane as is partially shown.

\section{Control Logic}

The NEA Scout flight control software (FCS) executes at a rate of $10 \mathrm{~Hz}$. During each iteration of the FCS, spacecraft attitude and angular rates are filtered, a phase-plane is evaluated for NEA Scout's three body axes, and pulse commands are sent to the RCS hardware. As described in the previous paragraphs, the phase-plane is a comparison of the current attitude and angular rate errors against the phase-plane switch lines. Like the more common Proportional-Integral-Derivative (PID) control logic, the phase-plane has several parameters that can be tuned to achieve desired performance. Some of these values are shown on the left of Figure 8 and were chosen for simplicity.

The values of $\pm \omega^{*}$ and $\pm \theta^{*}$ define the angular rate and attitude deadbands, respectively. These values define the $y$-intercept and $\mathrm{x}$-intercept for the sloped lines in the phase-plane. If desired, the upper and lower sloped lines could be defined individually, resulting in an asymmetric phase-plane with an additional two parameters required to tune. For simplicity, NEA Scout's phase-plane is symmetric and the lower sloped line is defined with the negative values of $\omega^{*}$ and $\theta^{*}$. The three parameters $\left(\omega^{*}, \theta^{*}\right.$, and $\left.\omega_{d}\right)$ are tuned by comparing fuel usage against response time for various maneuvers. This is done for each axis and each spacecraft configuration. For example, the phase-plane parameters when the solar sail is deployed are different than when it is stowed. The third parameter that defines the 
phase-plane is $\omega_{d}$. It defines the drift regions, which reduces propellant consumption for large attitude change maneuvers. Instead of continually firing the jets toward a sloped deadband, the drift regions stop the spacecraft and allow it to drift toward the desired state. Other tunable parameters are the pulse-width (chosen to be 50ms) and pulse duty cycle. When the solar sail is stowed, the duty cycle is the same rate as the FCS $(10 \mathrm{~Hz})$. After the solar sail is deployed, the RCS duty cycle is reduced to $0.7 \mathrm{~Hz}$ to limit flexible dynamic excitation. This is discussed in Section IV.C.

Referring to Figure 8, the three values $\left(\omega^{*}, \theta^{*}\right.$, and $\left.\omega_{d}\right)$ are used to define the phase-plane as shown in the following equations. Equation 1 describes the sloped switching lines. Here, slope is defined by the deadband values $\omega^{*}$ and $\theta^{*}$, and the y-intercept is the angular rate deadband $\omega^{*}$. The sign on the y-intercept designates either the upper or lower sloped line. In Equation 2, the value $\theta_{d}$ is calculated, which is the inflection point where the sloped lines become drift lines. Equation 3 is computed by substituting Eqn. 2 into Eqn. 1. Here, $\omega_{d o}$ designates the outer drift line, and the sign designates between the upper and lower lines.

$$
\begin{aligned}
& \omega=-1\left(\frac{\omega^{*}}{\theta^{*}}\right) \theta \pm \omega^{*} \\
& \pm \theta_{d}= \pm \frac{\omega_{d}+\omega^{*}}{\frac{\omega^{*}}{\theta^{*}}} \\
& \pm \omega_{d o}= \pm\left(\omega_{d}+2 \omega^{*}\right)
\end{aligned}
$$

A phase-plane is evaluated for each body axis during each iteration of the FCS. If-else statements are used to evaluate the phase-plane within the FCS. This is best described by the pseudo-code below, which is evaluated on each iteration of the FCS.

$$
\begin{aligned}
& \text { for } i=1: 3 \\
& \text { if } \theta_{i} \geq \theta_{d} \\
& \text { if } \omega_{i} \geq-\omega_{d} \\
& U_{i}=-1 \\
& \text { else if } \omega_{i} \leq-\omega_{d o} \\
& U_{i}=1 \\
& \text { (where } i \text { designates the } i^{\text {th }} \text { body axis) } \\
& \text { (attitude error on the } i^{\text {th }} \text { axis exceeds the value of } \theta_{d} \text { ) } \\
& \text { (angular rate error on the } i^{\text {th }} \text { axis exceeds the value of }-\omega_{d} \text { ) } \\
& \text { (command a negative torque on the } i^{\text {th }} \text { axis) } \\
& \text { else if } \theta_{i} \leq-\theta_{d} \\
& \text { if } \omega_{i} \leq \omega_{d} \\
& U_{i}=1 \\
& \text { else if } \omega_{i} \geq \omega_{d o} \\
& U_{i}=-1 \\
& \text { if } \omega_{i} \geq-1\left(\frac{\omega^{*}}{\theta^{*}}\right) \theta_{i}+\omega^{*} \\
& U_{i}=-1 \\
& \text { else if } \omega_{i} \leq-1\left(\frac{\omega^{*}}{\theta^{*}}\right) \theta_{i}-\omega^{*} \\
& U_{i}=1
\end{aligned}
$$

The first block evaluates whether or not the attitude error is to the right of $\theta_{d}$ in the phase-plane. If so, it then checks if the current angular rate error is outside the drift region. If so, the command torque for that axis is set to either positive or negative. This case would be triggered for the theoretical system trajectory shown on the right of Figure 8. Because the RCS system applies a fixed torque about each axis, only the sign of the torque is specified. The value of $U$ is used to select the correct jets to pulse, discussed in the following section. Similarly, the middle block of code evaluates whether or not the attitude error is to the left of $-\theta_{d}$ in the phase-plane. This block of code is mirrored from the first block, and evaluates if the current angular rate is outside the drift region in the upper-left quadrant of the phase-plane (Figure 8).

The bottom block of code handles the cases when the angular rate and attitude errors are near the sloped portion of the phase-plane. The first condition occurs when the attitude and angular rate errors are above the upper switching line. In this case, a negative torque is commanded on that axis to drive the system toward the deadband. The second 
condition represents the case when attitude and angular rate error are below the lower sloped line. In this case, a positive torque is commanded.

Last but not least, on each iteration of the for loop in the pseudo-code, a value is computed to determine the worst axis. In other words, the axis for which the attitude and angular rate errors are furthest away from the deadband. This axis is chosen for the current iteration of the FCS. In this way, only one axis is chosen per iteration of the FCS. This is a result of simplifying the control system and jet selection. Because only one pair of jets are fired at a time, only one spacecraft axis can be controlled per iteration of the FCS. On each iteration, the phase-planes are evaluated to determine the current worst axis, and two jets are chosen that provide torque on that axis. This is discussed in the following section.

\section{Jet Selection}

Simplicity was a major goal for the NEA Scout control system design. To that end, RCS jets are always fired in pairs based on the matrix shown in Table 1. This simplifies the control system as compared to a pulse-widthmodulation (PWM) scheme. The jet selection is performed as follows. First, the control logic outputs two values: the control axis and sign of the control torque. These values are then compared to the jet select matrix to determine which pair of jets to fire. For example, if the control logic determined that the $\mathrm{X}$-axis was worst, and was commanded a negative torque $(U=-1)$, jet's 2 and 3 would be fired (row 2 in the matrix). Compare this to Figure 6 , which shows the thrust directions of each RCS jet. It can be seen that firing these jets should produce a negative torque about the spacecraft's X-axis.

\begin{tabular}{c|cccc|ccc} 
Body Axis & Jet 1 & Jet 2 & Jet 3 & Jet 4 & Mx (N-mm) & My (N-mm) & Mz (N-mm) \\
\hline$+X$ & 1 & 0 & 0 & 1 & $\mathbf{3 . 5 3 4}$ & 0.007 & -0.008 \\
$-X$ & 0 & 1 & 1 & 0 & -3.159 & 0.007 & 0.008 \\
$+Y$ & 1 & 1 & 0 & 0 & 0.188 & $\mathbf{6 . 7 9 2}$ & 0.230 \\
$-Y$ & 0 & 0 & 1 & 1 & 0.188 & -6.778 & -0.230 \\
$+Z$ & 0 & 1 & 0 & 1 & 0.188 & 0.007 & $\mathbf{4 . 2 1 1}$ \\
$-Z$ & 1 & 0 & 1 & 0 & 0.188 & 0.007 & $-\mathbf{4 . 2 1 1}$
\end{tabular}

Table 1: Jet selection matrix used to select a particular pair of jets to fire to achieve a torque about a particular body axis. Values for torque have units of Newton millimeters.

In the table, the column on the left are the corresponding body axes and sign of the control torque to be applied to that axis. The middle columns designate the jets to be fired $(1=$ open, $0=$ close $)$. The columns on the right are the resulting torques (in Newton millimeters). These values were computed by taking the cross product of jet location and jet thrust vectors. Because the spacecraft's center of mass is not at the geometric center of the spacecraft, some cross-coupling results. For example, row 3 of the table shows the pair of jets used to produce a positive $\mathrm{Y}$-axis torque. While the $\mathrm{Y}$-axis moment is the largest, there are also some residual $\mathrm{X}$ and $\mathrm{Z}$-axis torques that occur. Similar crosscoupling exists when firing the $\mathrm{X}$ and $\mathrm{Z}$-axis jet pairs. Numerous simulations were run to ensure that cross-coupling is not a significant effect on the stability of the control system.

\section{RCS Sail Flex Avoidance Design}

After the solar sail is deployed, the RCS is used primarily for momentum desaturation. It is also used for any offnominal condition that requires attitude recovery. Due to their impulsive nature, the RCS pulses excite the flexible sail dynamics causing undesirable control system response. If the RCS responds to the sail dynamics, it can lead to limit cycling and high propellant use. To avoid exciting the sail dynamics, a $6^{\text {th }}$ order low pass filter with $0.1 \mathrm{~Hz}$ cutoff frequency is used in the attitude and body rate measurements of the RCS controller. Due to the high order of the filter, this introduces a significant lag in the measurements. The lag is so significant that, without compensation, it can destabilize the control system. Therefore, after the sail is deployed, the RCS pulse frequency is reduced from $10 \mathrm{~Hz}$ to $0.7 \mathrm{~Hz}$. In other words, the RCS jets pulse every 1.43 seconds instead of every 0.1 seconds. This ensures that the RCS does not overshoot the commanded rate and attitude during the filter lag time. The pulse frequency can be reduced further, but command tracking performance degrades due to the slow response of the spacecraft. Therefore, $0.7 \mathrm{~Hz}$ was chosen to provide desirable response time and minimize propellant usage, while also maintaining stability. This is discussed in Section V.D. 


\section{Momentum Wheel Desaturation Design}

NEA Scout is controlled by reaction wheels for most of its mission. Environmental torques - especially those caused by the solar sail - cause momentum accumulation on the reaction wheels. The solar sail torque is simply referred to as solar torque, and is mostly due to solar sail thrust not acting through the spacecraft's CM. The attitude control system continually responds to those disturbance torques resulting in reaction wheel speed buildup. This buildup must be managed to ensure the reaction wheels have enough capacity to control the spacecraft. Actively controlling the reaction wheel momentum is referred to as momentum management and wheel desaturation.

On NEA Scout, reaction wheel momentum is managed differently depending on the mission phase and spacecraft configuration. Before sail deployment, wheel momentum is managed entirely by the RCS, described in the following paragraph. After sail deployment, the AMT manages X and $\mathrm{Y}$-axis momentum, leaving Z-axis momentum management to the RCS. Again, the AMT manages momentum by shifting the spacecraft's CM relative to the sail's center of pressure (CP). This effect is visualized by the graphic in Figure 9 below. In this way, the solar torque can either be trimmed or reversed to desaturate the wheels. For redundancy, the RCS is reserved as a backup actuator for $\mathrm{X}$ and $\mathrm{Y}$-axis momentum management throughout the mission.

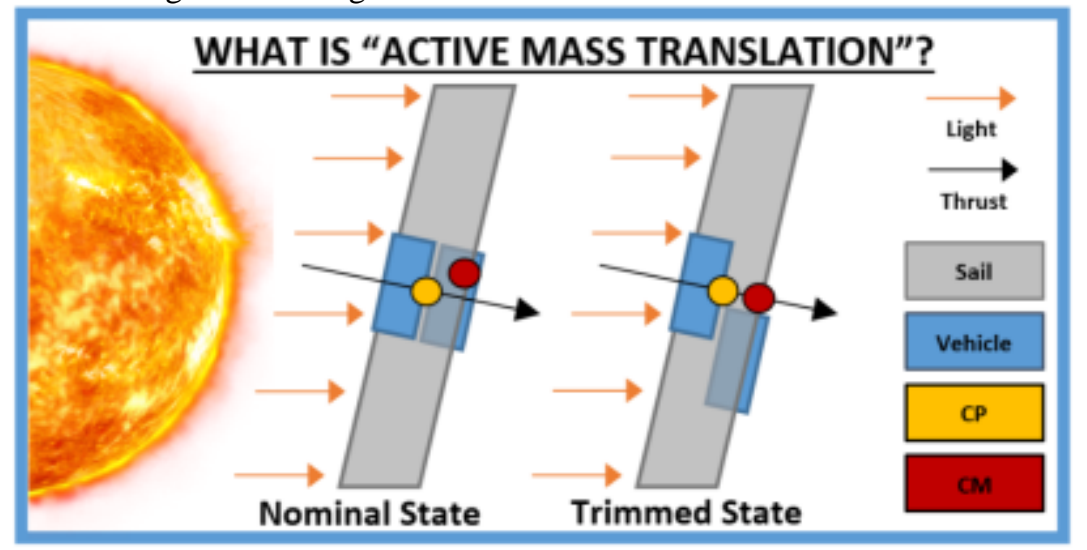

Figure 9: Graphic depicting the effect of shifting the Center of Mass $(\mathrm{CM})$ relative to the Center of Pressure (CP). The AMT can shift the CM enough to both change the direction of solar torque, or trim the solar sail to achieve no solar torque.

RCS momentum management works by monitoring the momentum buildup on each of the four wheels. The momentum of each wheel is first transformed to compute the momentum on the spacecraft body axes. When the momentum exceeds a specified threshold, the flight control system commands the RCS to apply a torque about the body axis with the largest momentum. To provide a torque, RCS jets are chosen based on the Jet Selection Matrix as shown in section IV.B.2. The RCS controller executes the commanded torque by pulsing the jets at a particular duty cycle and pulse width. The resulting torque is perceived by the attitude control system as a disturbance, causing the reaction wheels to respond to the disturbance and driving the wheel speeds toward zero. The RCS pulse width and duty cycle are carefully tuned to not overwhelm the reaction wheel attitude controller. Desaturation is reached when the momentum on each axis falls below a specified deadband. During a slew, the momentum is biased to include the product of commanded slew-rate and spacecraft inertia. This bias allows the momentum management system to assist the reaction wheels in tracking the required slew rates.

\section{RCS Control Performance}

\section{A. Initial Detumble and Sun Pointing}

As mentioned in Section IV.A, the first responsibility of the RCS is to detumble the spacecraft and point to the sun for charging. In the worst case, NEA Scout will be ejected with angular rates of $16 \%$ s about each body axis. As soon as NEA Scout powers on, the RCS will null those rates and immediately point its solar panels toward the sun for charging. For simplicity, these maneuvers are performed in series - first detumble then sun pointing. Figure 10 shows simulated results of the detumble maneuver with angular rates on the left and propellant usage on the right. Here, the initial angular rates are $16 \%$. Using the onboard IMU to measure angular rates, RCS control is engaged at $t=20 \mathrm{~s}$ and it quickly nulls the rates to within the angular rate deadband $(0.2 \%)$. The angular rate oscillations during the initial coast period is dynamic precession, the effect of having a non-diagonal inertia matrix. The right of Figure 10 shows that NEA Scout used about three grams of propellant during the maneuver. 

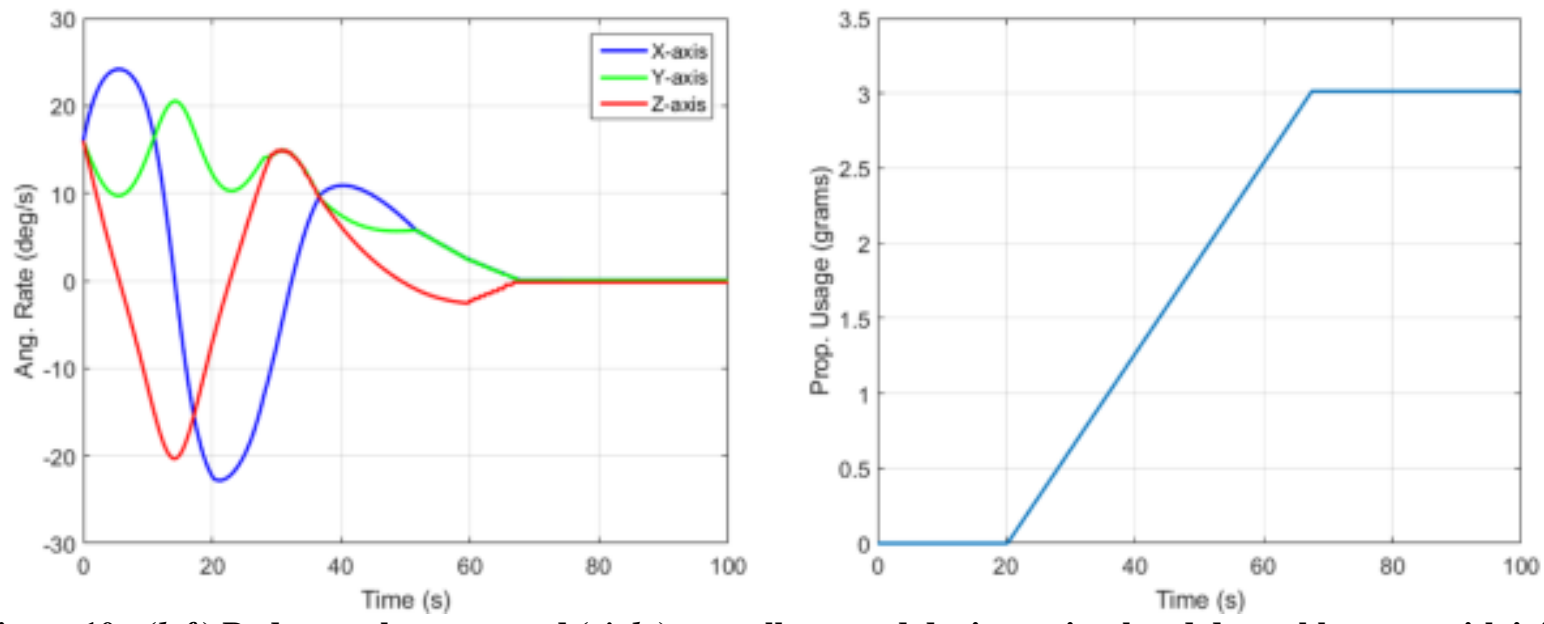

Figure 10: (left) Body angular rates and (right) propellant used during a simulated detumble event with initial rates of $16 \%$ about each axis. RCS control is engaged at $t=20$ s to null the spacecraft angular rates.

After detumble, NEA Scout must point its solar panels toward the sun for charging. To do this, it utilizes its three sun sensors described in Section III.1. After detumble, there is a possibility that none of the sun sensors will be in view of the sun. In this case, an autonomous sun-finding maneuver must be performed. Here, a 90 degree slew is commanded about the $\mathrm{Y}$-axis, followed by a 90 degree slew about the $\mathrm{X}$-axis. If the sensors find the sun at any time during the slews, the pre-programmed maneuver is interrupted. This is a worst case scenario because it will take the longest time to point toward the sun before charging. Figure 11 shows the commanded versus actual angular rates during this maneuver. As shown, the RCS phase-plane controller is able to follow the commanded slew pattern in order to find the sun. The total sun finding maneuver takes about 450 seconds. Figure 12 provides the propellant used during the sun-finding maneuver, requiring less than a gram of propellant to find the sun.
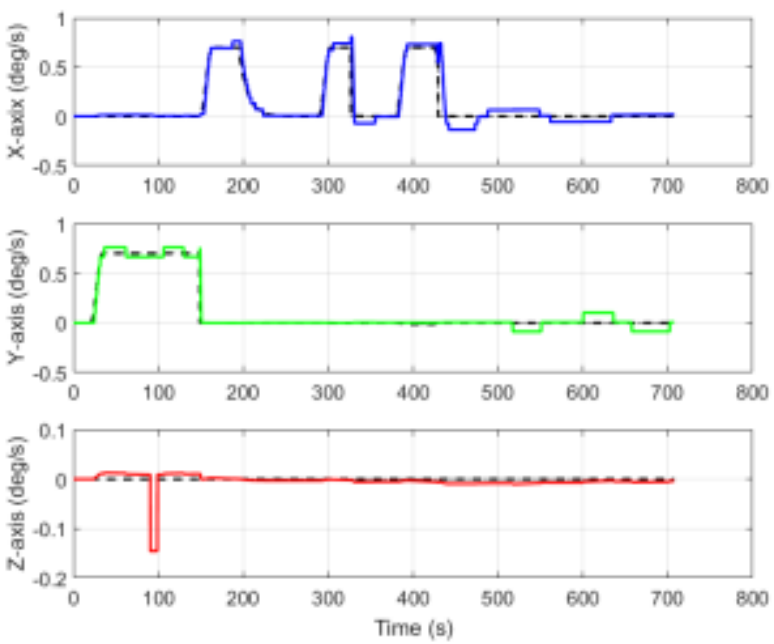

Figure 11: Commanded (dashed lines) vs. actual angular rates (solid) during an autonomous sun-finding maneuver. 


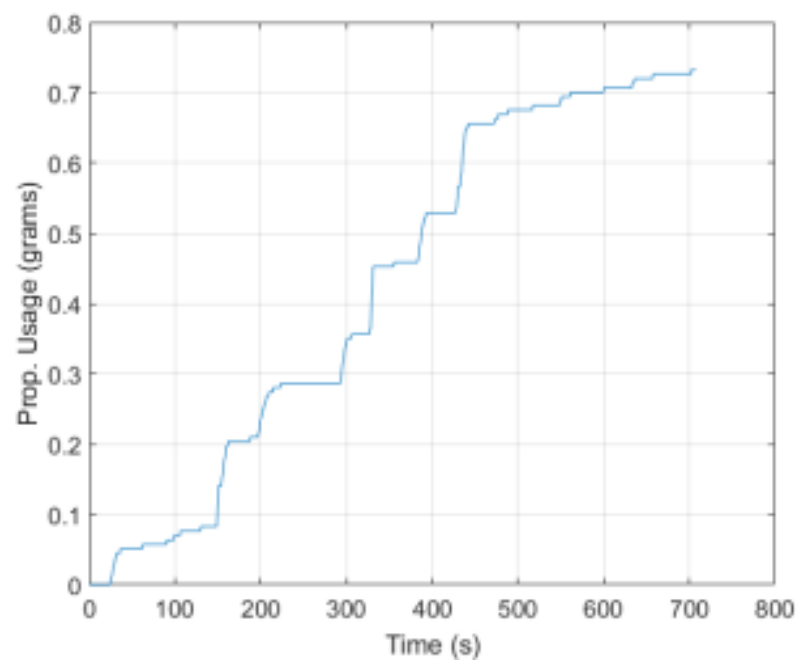

Figure 12: Propellant used during a sun-finding maneuver to initially point the spacecraft towards the sun for charging.

\section{B. Trajectory Correction Maneuver}

Another responsibility of the RCS is the Trajectory Correction Maneuver (TCM). This maneuver is discussed in Section IV.A and is performed to achieve a particular lunar fly-by. During this maneuver, the RCS axial jets are opened to provide thrust $(\boldsymbol{\Delta V})$. At the same time, the RCS attitude control jets are pulsed to control attitude and angular rates. Furthermore, to conserve propellant the axial jets are off-pulsed during the maneuver to supplement $\mathrm{Y}$ axis control. Due to power and propellant heating constraints, the TCM is performed in 10-minute segments. Several segments will be performed to achieve the desired total $\boldsymbol{\Delta} \boldsymbol{V}$. Figure 13 shows the results from a simulated TCM segment between $t=100 \mathrm{~s}$ and $t=700 \mathrm{~s}$.
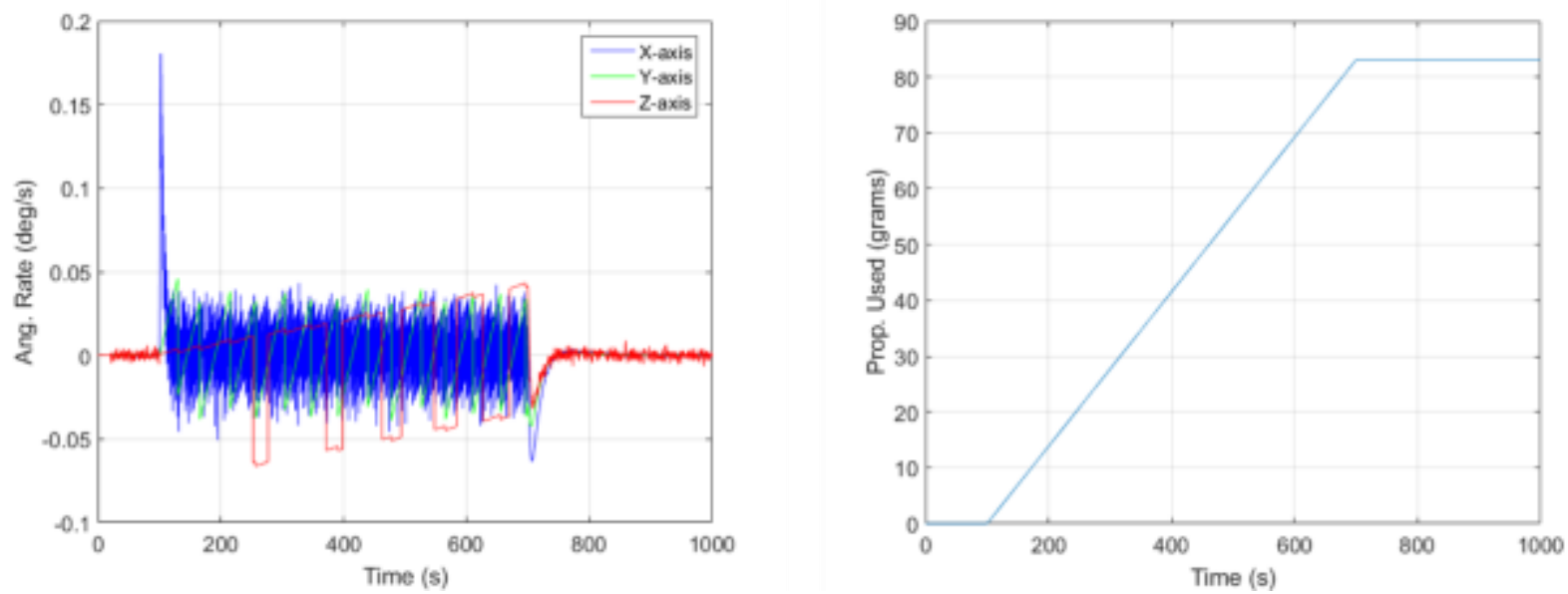

Figure 13: (left) Body angular rates and (right) propellant usage during a 10-minute TCM segment from $t=100$ to $t=700$ seconds.

The angular rates are shown on the left and propellant usage is on the right. The angular rates show that the Xaxis is by far the most active, with $\mathrm{Y}$ and Z-axis pulses occurring less frequently. The reason for the high activity on the $\mathrm{X}$-axis is due to the location of the center of mass (CM) relative to the axial jets. Referring to Figure 6, the location of the CM is slightly above the vehicle centerline (offset along the $\mathrm{Y}$-axis). Therefore, the axial jets produce a small $\mathrm{X}$-axis disturbance torque that the attitude control jets must constantly correct. Even with the high pulse count on the $\mathrm{X}$-axis jets, the propellant used during the segment is about 83 grams. The best case scenario (i.e. no attitude control) would result in 77 grams of propellant used. Therefore, the propellant cost of attitude control during this segment is roughly 6 grams. 


\section{Momentum Wheel Desaturation}

Figure 14 and Figure 15 show the results of the RCS momentum management controller. Initially, the wheel momentum exceeds the threshold in all three body axes. For this case study, the spacecraft configuration is pre-sail deployment with only the solar arrays deployed (Figure 3, left). In this configuration, the spacecraft inertias are about 64 times less than when the sail is deployed. Therefore, the RCS firings produce large angular accelerations (i.e. large disturbances) to which the wheels must respond. This configuration is shown because it results in a stressing case. First, RCS pulses produce attitude disturbances much faster than when the sail is deployed. Second, the reaction wheel controller must respond fast enough to absorb those attitude disturbances to desaturate without allowing the spacecraft to experience large attitude displacements.

Figure 14 shows the commands sent to the RCS to open the jets. The dependent axis is the pulse width (in seconds). As shown, the commanded pulse widths are $50 \mathrm{~ms}(0.05 \mathrm{~s})$ in duration. The independent axis shows the simulation time. In other words, the commands sent to each jet are pulse widths, commanded at particular times during the simulation. Jet's 5 and 6 are the axial jets, which are not used for attitude control and are zero throughout the simulation. Figure 15 shows the response of reaction wheel speeds to RCS pulses, which successfully reduces the wheel speeds within the controller deadband.

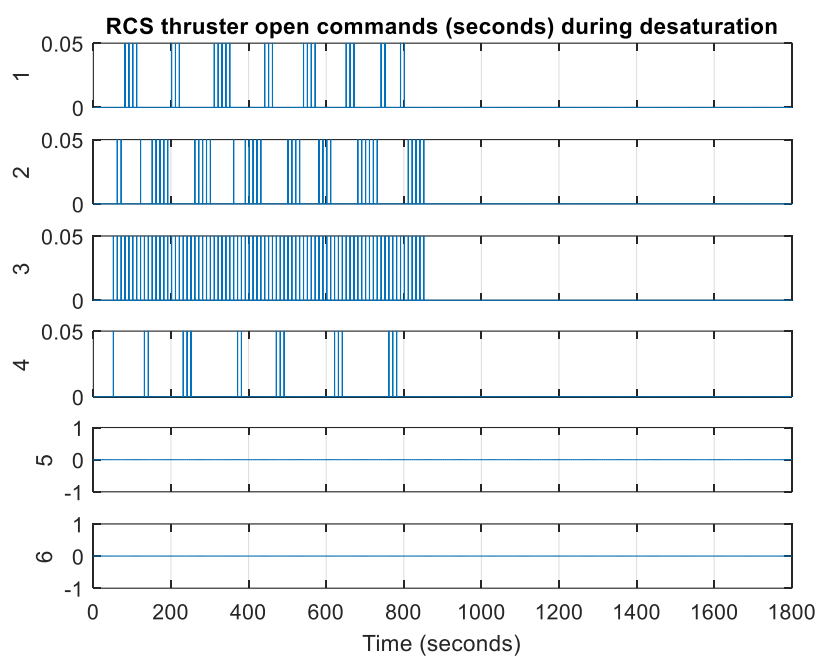

Figure 14: RCS thruster firing commands during a reaction wheel desaturation event when the momentum threshold is exceeded in all three body axes. The commands are pulse widths sent at particular times during the simulation. This is in the pre-sail deployed configuration when the spacecraft moments of inertia are lowest.

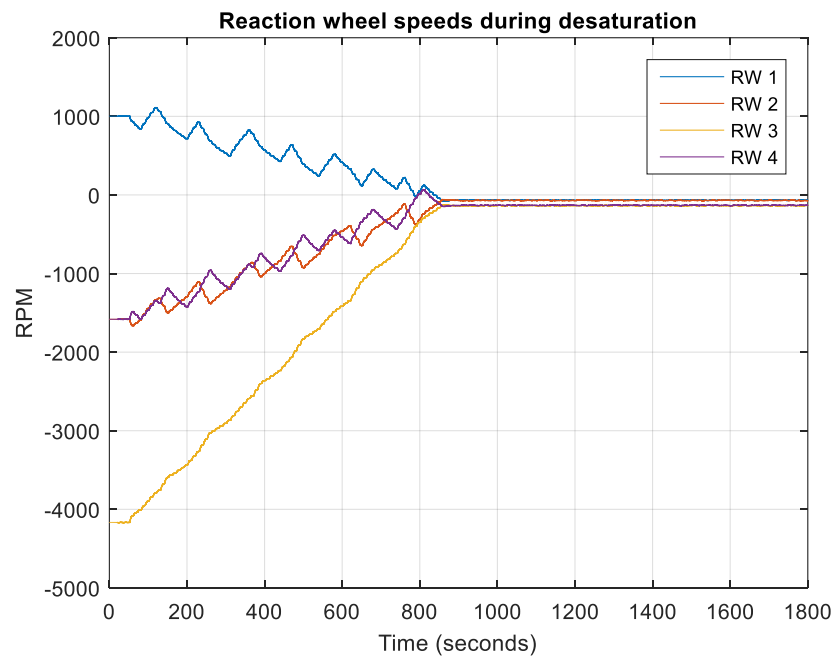

Figure 15: Reaction wheel speeds when using the RCS for desaturation. Initially, the momentum threshold is exceeded in all three body axes. The spacecraft is in the pre-sail deployed configuration when moments of inertia are low. 


\section{Sail Flex Dynamics}

Due to their impulsive nature, RCS pulses will excite all the flexible modes of the spacecraft. This is of particular concern for a solar sail spacecraft, which typically have low frequency flex modes associated with the sail. When those modes are excited, the RCS controller will respond to counteract them. This results in high jet pulse cycling and associated high propellant usage. Under RCS control, Figure 16(a) shows the body rate response to a commanded 90 degree slew and subsequent attitude hold after $t=1500$ s. Figure 16(b) shows the associated propellant usage during that time (about 300 grams). In this simulation, the RCS controller is responding to the flexible dynamics, resulting in a limit cycling behavior (constantly overshooting the RCS deadbands). This leads to high frequency spacecraft body rates and high propellant usage. However, as discussed in Section IV.C, a low pass filter was added to the measured angular rates to remove the flex oscillations from the angular rates. This, combined with a reduced pulse frequency, was implemented to avoid responding to the sail flexible dynamics. Figure 17(a) shows the spacecraft's response to the same slew and attitude hold once the filter and reduced pulse frequency are applied. Similarly, Figure 17(b) shows the associated propellant consumption. Comparing Figure 16 and Figure 17 shows that the propellant usage fell from approximately 310 grams to less than 2 grams. The propellant savings allows for increased margins for any off-nominal or safe mode operations. This design also reduces total open-close cycles of the RCS valves, therefore extending the life span of the system.

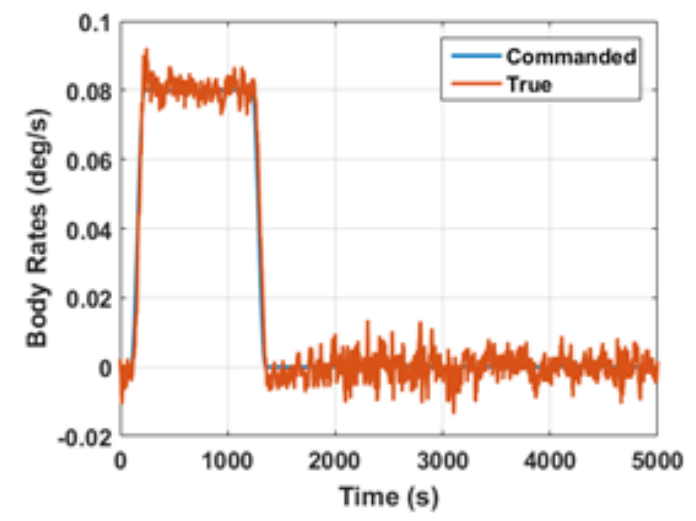

(a)

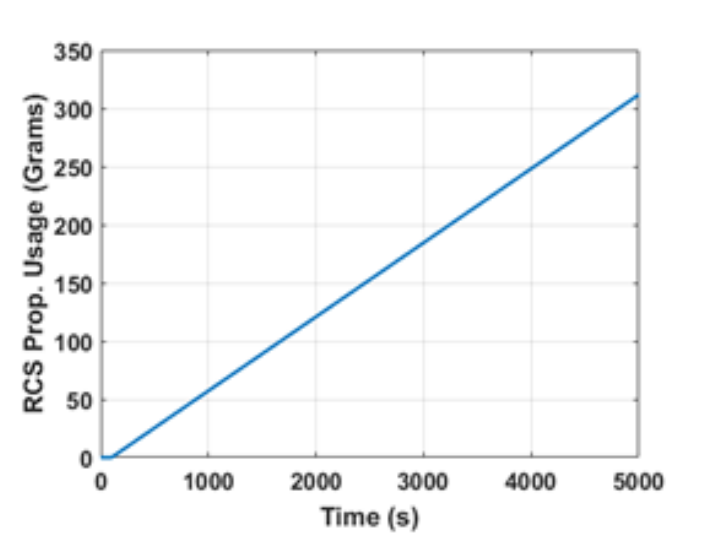

(b)

Figure 16: (a) Commanded vs. actual body rates and $(b)$ RCS propellant usage during a simulated 90 degree Y-axis slew maneuver and subsequent attitude hold without filtering flexible modes.

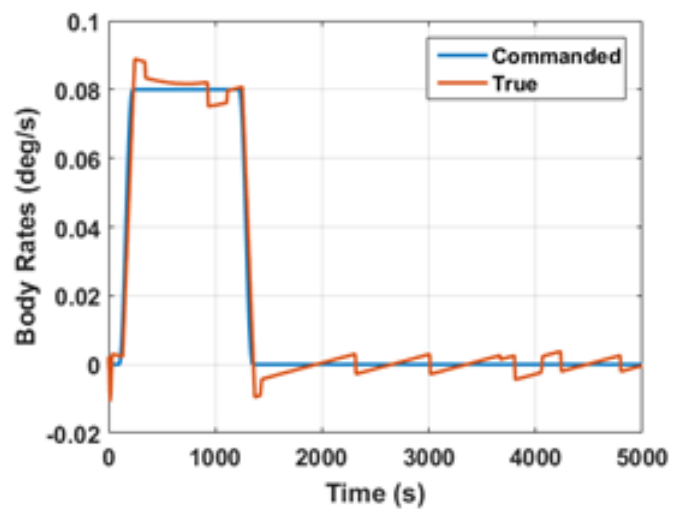

(a)

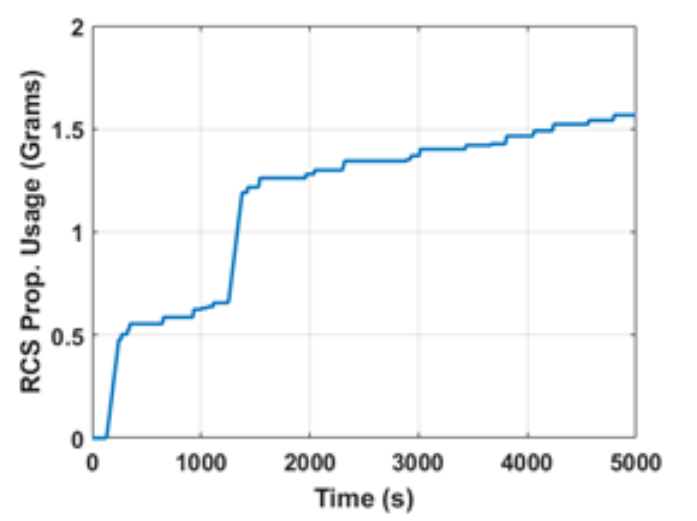

(b)

Figure 17: Commanded vs. actual body rates $(a)$ and RCS propellant usage $(b)$ during a simulated 90 degree slew maneuver and subsequent attitude hold with filtering and reduced RCS pulse frequency.

\section{Conclusions}

This paper provides an overview of the NEA Scout CubeSat's attitude control system. It particularly focuses on the cold gas Reaction Control System (RCS), described in Section III.A.1. The unit is being manufactured by VACCO Industries, and uses refrigerant R236fa for propellant. It has four attitude control jets used to impart torque, and two 
axial jets used to impart thrust onto the spacecraft. The RCS is the primary control actuator during several portions of NEA Scout's mission. In particular, it performs the initial spacecraft detumble (null rates) and points toward the sun after NEA Scout is ejected. It must also perform a trajectory correction maneuver (TCM), and desaturate the reaction wheels throughout the mission. Simulated results of these events are discussed in Section V and show the performance of the control system at meeting these requirements. In order to control the spacecraft, the RCS uses a novel but simple control logic known as a phase-plane. This is combined with a very simple jet-select logic, where jets are always fired in pairs to generate torque about the three spacecraft body axes. This is described in Section IV. This section also describes how the control system is designed to avoid exciting NEA Scout's flexible dynamics, and also how the RCS is used for momentum management. The control systems performance is shown in Section V. Here, time-domain plots are shown of various simulated events, showing NEA Scout's performance against several worst-case requirements. These plots show the RCS ability to track commanded slew patterns, and how it avoids exciting sail dynamics. Propellant usage is also shown during these maneuvers, since propellant is a driving constraint in the design of an RCS control logic. Most maneuvers use about 5 grams of propellant, while some require less than a gram. This is a significantly low percentage of total propellant $(1.25 \mathrm{~kg})$ per maneuver. Based on numerous simulations, it is believed that NEA Scout's RCS control logic provides a robust and efficient control system to meet the NEA Scout mission objectives.

\section{References}

1 NASA JPL Small-Body Database Browser, 1991 VG. Link: https://ssd.jpl.nasa.gov/sbdb.cgi?sstr=1991VG.

2 McNutt L., Johnson L., Kahn P., Julie Castillo-Rogez, and Andreas Frick: Near-Earth Asteroid (NEA) Scout, 20144435, AIAA SPACE Conference and Expo, San Diego, CA, 2014.

3 Vinh Bach et al.: Near Earth Asteroid (NEA) Scout Solar Sail Implementation, Annual AIAA/USU Conference on Small Satellites; 30th; 6-11 Aug. 2016; Logan, UT; United States, 2016, available at: https://ntrs.nasa.gov/search.jsp?R=20160011083.

4 Johnson, L., "Near Earth Asteroid (NEA) Scout", The Fourth International Symposium on Solar Sailing, 170002, ISSS, Kyoto, Japan, 2017. Available at http://www.jsforum.or.jp/ISSS2017/papers/index.html.

5 Orphee, J., "Solar Sail Attitude Control System for the NASA Near Earth Asteroid Scout Mission", The Fourth International Symposium on Solar Sailing, 17091, ISSS, Kyoto, Japan, $2017 . \quad$ Available at http://www.jsforum.or.jp/ISSS2017/papers/index.html.

6 Bryson, A. E., Control of Spacecraft and Aircraft, Princeton University Press, New Jersey, 1994, pp. 34-36.

7 Wertz, J. R. (Ed.), Spacecraft Attitude Determination and Control, Reidel Publishing Company, Holland, 1978.

8 Wie, B, Space Vehicle Dynamics and Control, American Institute of Aeronautics and Astronautics, Virginia, 1998.

9 Sidi, M. J., Spacecraft Dynamics and Control: A Practical Engineering Approach, Cambridge University Press, New York, 1997. 